UTHEP-452

UTCCP-P-117

\title{
Non-perturbative renormalization for a renormalization group improved gauge action *
}

\author{
CP-PACS Collaboration:
}

S. Aoki, ${ }^{a}$ R. Burkhalter, ${ }^{a}, \mathrm{~b}$ M. Fukugitac ${ }^{c}$ S. Hashimoto, ${ }^{\mathrm{d}}$ K. Ide, ${ }^{\mathrm{a}}$ N. Ishizuka, ${ }^{\mathrm{a}, \mathrm{b}}$ Y. Iwasakia,b K. Kanaya, T. Kaneko, ${ }^{\mathrm{d}}$ Y. Kuramashi, V. Lesk, M. Okawa, ${ }^{\mathrm{d}}$ Y. Taniguchi, ${ }^{\mathrm{a}}$ A. Ukawa ${ }^{\mathrm{a}, \mathrm{b}}$ and T. Yoshié ${ }^{\mathrm{a}, \mathrm{b}}$

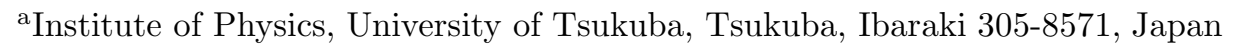

${ }^{\mathrm{b}}$ Center for Computational Physics, University of Tsukuba, Tsukuba, Ibaraki 305-8577, Japan

${ }^{\mathrm{c} I n s t i t u t e}$ for Cosmic Ray Research, University of Tokyo, Kashiwa, Chiba 277-8582, Japan

${ }^{\mathrm{d}}$ High Energy Accelerator Research Organization (KEK), Tsukuba, Ibaraki 305-0801, Japan

\begin{abstract}
Renormalization constants of vector $\left(Z_{V}\right)$ and axial-vector $\left(Z_{A}\right)$ currents are determined non-perturbatively in quenched QCD for a renormalization group improved gauge action and a tadpole improved clover quark action using the Schrödinger functional method. Non-perturbative values of $Z_{V}$ and $Z_{A}$ turn out to be smaller than the one-loop perturbative values by $O(10 \%)$ at $a^{-1} \approx 1 \mathrm{GeV}$. A sizable scaling violation of meson decay constants $f_{\pi}$ and $f_{\rho}$ observed with the one-loop renormalization factors remains even with non-perturbative renormalization.
\end{abstract}

\section{Introduction}

Reliable lattice calculations of hadronic matrix elements and quark masses require both high precision numerical simulations and nonperturbative determinations of renormalization constants ( $Z$-factors). The CP-PACS collaboration recently carried out a sophisticated spectrum calculation in $N_{f}=2$ full QCD [1] using a renormalization group (RG) improved gauge action and a tadpole improved clover quark action. However, non-perturbative $Z$-factors were not available for this action combination. Hence analyses had to rely on one-loop perturbative values.

As a first step toward a systematic study of non-perturbative renormalization for this action, we apply the Schrödinger functional method [2] to calculations of $Z$ factors for vector $\left(Z_{V}\right)$ and axial-vector $\left(Z_{A}\right)$ currents in quenched QCD with the same improved action. We examine in particular whether a large scaling violation of meson decay constants observed for this action [1] is improved with non-perturbative $Z$-factors. We report preliminary results in these proceedings.

\section{Calculational Method}

We follow the method developed by the ALPHA collaboration [3]. Namely, we use a lattice geometry of $L^{3} \cdot T$ with $T=2 L$ for $Z_{V}$ with a vector operator at $t=L$, and $T=3 L$ for $Z_{A}$ with two axial vector operators at $t=L$ and $t=2 L$, except at $\beta=2.2$ and 2.4 for $Z_{A}$ (see sec. 3 for details of this exception). Tree-level values are used for coefficients of boundary counter terms of the action. For improving the axial current, we use the one-loop perturbative value for the coefficient $c_{A}$.

Values of $Z_{V}$ and $Z_{A}$ are determined for $\beta=$ $2.2-8.0$ which almost covers the range of the CP-PACS quenched spectrum calculation [1], $\beta=$ $2.187-2.575$. Physical size is normalized at $\beta=$ 2.6 on an $8^{3}$ lattice. For other $\beta$ values, two lattice sizes are analyzed to match the physical size using the string tension. Our action has $O(a)$ errors since we employ a tadpole improved value of $c_{\mathrm{sw}}=(1-0.8412 / \beta)^{-3 / 4}$. Therefore we extrapolate/interpolate results linearly in $1 / L$. We have analyzed 300-4000 configurations depending on $\beta$ value and lattice size.

\footnotetext{
*Talk presented by K. Ide.
} 


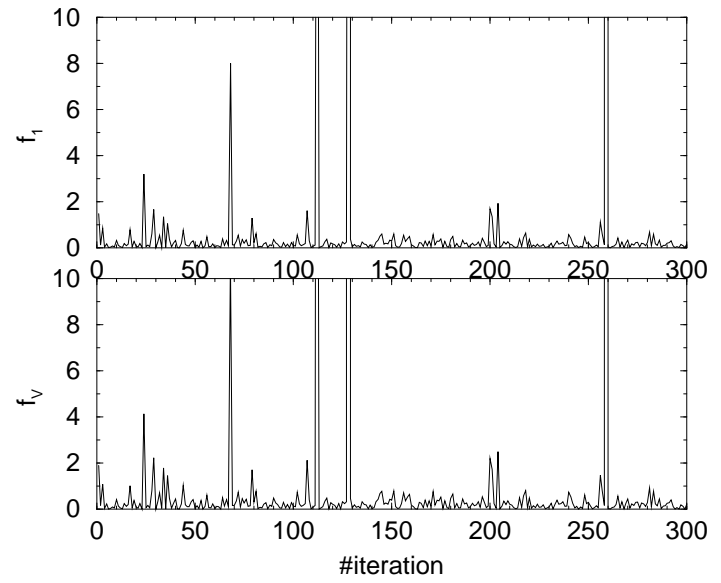

Figure 1. Time history of $f_{1}$ and $f_{V}$ at $\beta=2.4$ on an $8^{3} \times 16$ lattice.

\section{Exceptional Configurations}

It is straight-forward to calculate $Z$-factors for $\beta>2.4$ for $Z_{V}$ and $\beta>2.8$ for $Z_{A}$. We find reasonable plateaux in the ratio of Green functions for the $Z$-factors in spite of the $O(a)$ error of the action, which implies viability of the Schrödinger functional method for our action.

However, for lower $\beta$ values on a large lattice, anomalously large values appear in the ensemble of $f_{1}, f_{V}$ and $f_{A A}$ where $Z_{V}=f_{1} / f_{V}$ and $Z_{A}=$ $\sqrt{f_{1} / f_{A A}}$. This is illustrated with a time history of $f_{1}$ and $f_{V}$ at $\beta=2.4$ in Fig. 且.

In order to estimate $Z$-factors at low $\beta$ values, we have investigated the properties of these "exceptional configurations". We find : i) Large values of $f_{1}$ and $f_{V}$ for $Z_{V}$ and $f_{1}$ and $f_{A A}$ for $Z_{A}$ are strongly correlated (see Fig. 1). ii) Histograms of $f$ 's have a long tail toward very large values as shown in Fig. 2. We then impose a cutoff in taking the average of the $f$ 's, and find that $Z$ factors are stable against change of the cutoff as long as anomalously large values are discarded, as the numerator and denominator for $Z$-factors are correlated and effects mostly cancel out. See Fig. 3.

We then estimate $Z$-factors for low $\beta$ values taking a certain value of the cutoff. For $Z_{A}$ at $\beta=2.2$ and 2.4 , the lattice geometry is also changed from $T=3 L$ to $T=2 L$ because "exceptional configurations" appear very frequently

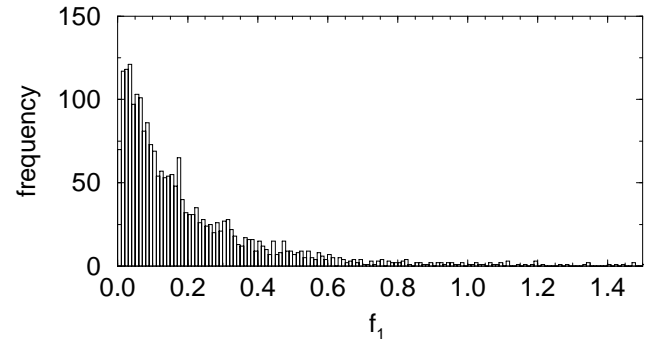

Figure 2. Histogram of $f_{1}$ at $\beta=2.4$ on an $8^{3} \times 16$ lattice.

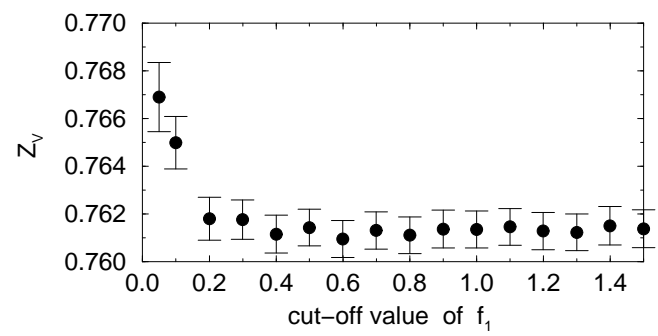

Figure 3. Values of $Z_{V}$ estimated by discarding configurations with $f_{1}$ larger than the cutoff value. Data are for an $8^{3} \times 16$ lattice at $\beta=2.4$.

for the original geometry to the extent that the cutoff analysis above does not work. We have checked at $\beta=2.6$ that the change of geometry does not lead to any significant difference in $Z_{A}$.

\section{Results for $Z$-factors}

In Fig. A we show results of $Z$-factors together with Padé fits (solid curves in the figure) to them. Non-perturbative estimates give values smaller than the one-loop perturbative ones (dashed lines) by about $10 \%(6 \%)$ for $Z_{V}\left(Z_{A}\right)$ at the largest coupling of the CP-PACS simulation, $\beta=2.187$.

\section{Scaling Property of Decay Constants}

We compare in Fig. $5 f_{\pi}$ and $f_{\rho}$ determined with non-perturbative (filled circles) and perturbative (open circles) $Z$-factors. Also shown are the results from the standard plaquette and Wilson action (squares) 4] using the perturbative $Z$ factors.

We observe that, even with the non-perturbative $Z$-factors, large scaling violation of meson decay constants remains for the range we have in- 

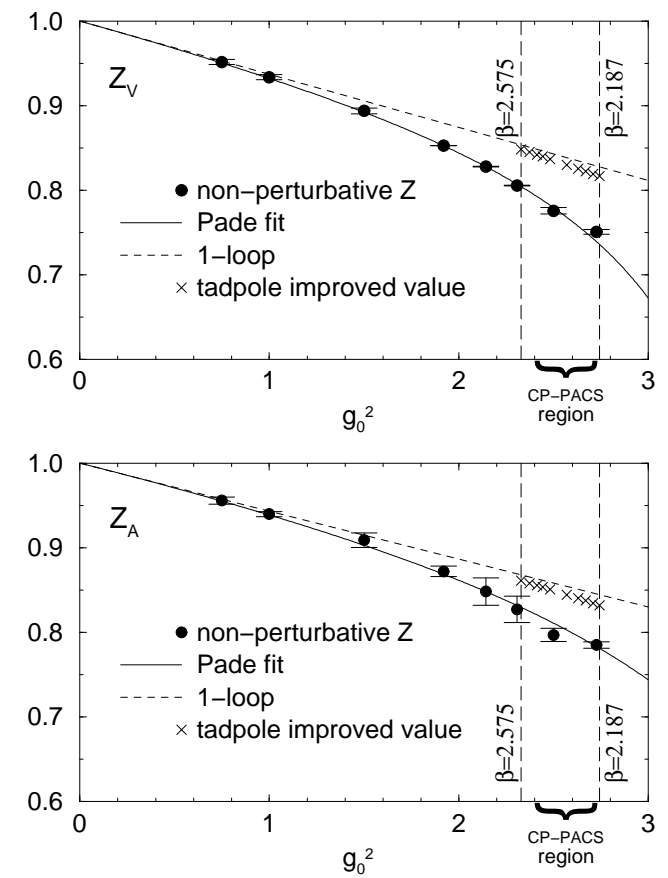

Figure 4. Results for $Z_{V}$ (top) and $Z_{A}$ (bottom).

vestigated. A possible reason is the necessity of non-perturbatively fixing the $O(a)$ and perhaps higher terms in the currents themselves. For the axial vector current, it will be worth investigating if non-perturbative estimates of the $O(a)$ coefficient $c_{A}$ yield a large value.

\section{Conclusions}

We have successfully applied the Schrödinger functional method to calculations of $Z_{V}$ and $Z_{A}$ for the combination of a RG-improved gauge action and a tadpole improved clover quark action down to the lattice spacings $a^{-1}=1-2 \mathrm{GeV}$ where the quenched CP-PACS data for decay constants were taken.

While $Z$-factors estimated non-perturbatively are smaller by $O(10 \%)$ than perturbative ones for this range, there still remain large scaling violations of $O(a)$ and higher in meson decay constants with non-perturbative $Z$-factors. Further work is needed to examine if hadronic matrix elements could be reliably extracted at lattice spacings much coarser than $a^{-1} \approx 2 \mathrm{GeV}$ with op-
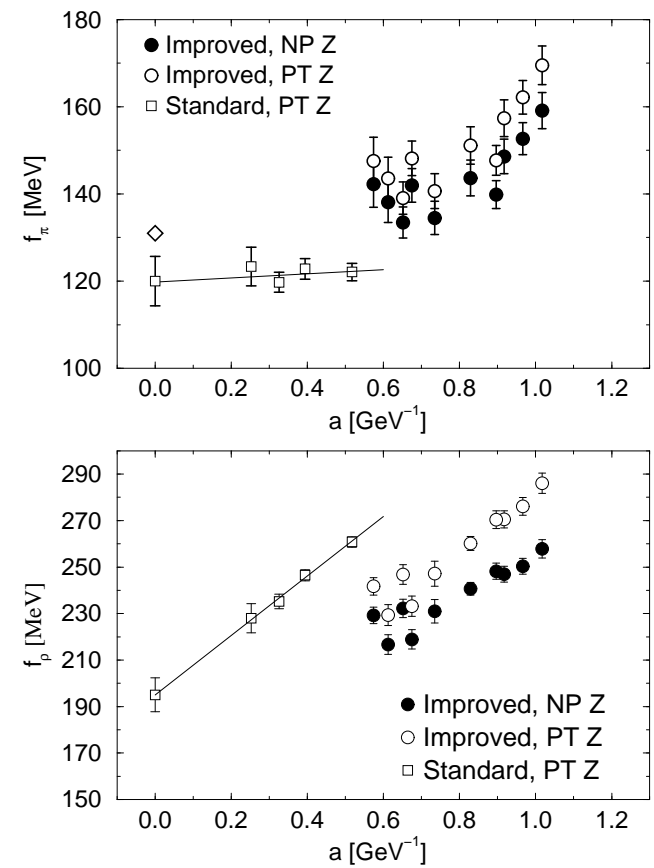

Figure 5. $f_{\pi}$ (top) and $f_{\rho}$ (bottom) vs. $a$ for our improved action with non-perturbative (NP) and perturbative (PT) $Z$-factors together with results for the standard action 迎.

erators improved non-perturbatively at $O(a)$ and beyond.

This work is supported in part by Grants-inAid of the Ministry of Education (Nos. 10640246, 10640248, 11640250, 11640294, 12014202, 12304011, 12640253, 12740133, 13640260 ). VL is supported by the Research for Future Program of JSPS (No. JSPS-RFTF 97P01102).

\section{REFERENCES}

1. CP-PACS collaboration: A. Ali Khan et al., hep-lat/0105015.

2. M. Lüscher et al., Nucl. Phys. B384 (1992) 168.

3. M. Lüscher et al., Nucl. Phys. B491 (1997) 344.

4. CP-PACS collaboration: S. Aoki et al., Phys. Rev. Lett. 84 (2000) 238. 